\section{Physikalisch-Technische Bundesanstalt}

T. Arndt

Bioscientia Institut für Medizinische Diagnostik $\mathrm{GmbH}$, Ingelheim, Deutschland

\section{Synonym(e) PTB}

Englischer Begriff Physikalisch-Technische Bundesanstalt

Definition Nationales Metrologie-Institut mit wissenschaftlich-technischen Dienstleistungsaufgaben und wissenschaftlich-technische Bundesoberbehörde im Geschäftsbereich des Bundesministeriums für Wirtschaft und Technologie.

Beschreibung Die PTB wurde im Jahr 1887 als Physikalisch-Technische Reichsanstalt auf Initiative und nach Ideen von Werner von Siemens (1816-1892) und Hermann von Helmholtz (1821-1894) gegründet. Standorte sind Braunschweig und Berlin-Charlottenburg. In neun wissenschaft- lich-technischen Abteilungen (davon zwei in Berlin) mit ca. 60 Fachbereichen und rund 200 Arbeitsgruppen arbeiten etwa 1900 Beschäftigte. Die PTB betreibt Grundlagenforschung und Entwicklung in faktisch allen Bereichen der Physik und Messtechnik. Im Einheiten- und Zeitgesetz werden der PTB die Darstellung und die Weitergabe der Einheiten überantwortet. Am aktuellen Umbau des Einheitensystems ist die PTB maßgeblich beteiligt: etwa mit dem AvogadroProjekt zur Neudefinition von Kilogramm und Mol, dem Projekt „Boltzmann-Konstante“ zur Neudefinition des Kelvin und bei dem Versuch, das Ampere auf die Elementarladung des Elektrons zurückzuführen. Die Weiterentwicklung von Messmethoden und Messverfahren in der Medizin ist ein Arbeitsgebiet der derzeitigen Abteilung 8 .

\section{Adresse:}

Physikalisch-Technische Bundesanstalt (PTB)

Bundesallee 100

D-38116 Braunschweig

Tel.: 0531592.

www.ptb.de 